\section{Fredrickson, Donald S.}

K. J. Lackner ${ }^{1}$ und D. Peetz ${ }^{2}$

${ }^{1}$ Institut für Klinische Chemie und Laboratoriumsmedizin, Universitätsmedizin Mainz, Mainz, Deutschland

${ }^{2}$ Institut für Labormedizin, Helios Klinikum Berlin-Buch, Berlin, Deutschland

Lebensdaten Amerikanischer Physiologe, geboren am 8. August 1924 in Canon City, Colorado, USA, gestorben am 7. Juni 2002 in Bethesda, Maryland, USA.
Verdienste Fredrickson hat sich wissenschaftlich mit Erkrankungen des Fettstoffwechsels und deren Beziehung zur Atherosklerose beschäftigt. $\mathrm{Er}$ entwickelte in den 1960er-Jahren des letzten Jahrhunderts eine Klassifikation der Hyperlipidämien, die inzwischen weitgehend durch eine stärker pathophysiologisch orientierte Betrachtung abgelöst wurde ( $\triangleright$ Fredrickson-Klassifikation). 